\title{
INTEGRAÇÃO DE FERRAMENTAS DO DESIGN THINKING E DA METODOLOGIA TRIZ NA FASE CONCEITUAL DE UM PROCESSO DE DESENVOLVIMENTO DE UM NOVO PRODUTO NA INDÚSTRIA AUTOMOTIVA.
}

\author{
Ricardo Henrique da Silva, Prof. Dr. Paulo Carlos Kaminski \\ Universidade de São Paulo, Escola Politécnica \\ E-mails: ricardohsilva@usp.br, pckamins@usp.br
}

\begin{abstract}
RESUMO
O objetivo desta pesquisa será o de investigar se metodologias de inovação como o Design Thinking (DT) e a metodologia TRIZ podem ser aplicadas de maneira sinérgica ao tradicional modelo de APQP (Advanced Product Quality Planning) utilizado pela indústria automotiva no desenvolvimento de novos produtos. Para atender tal objetivo buscou-se entender dentro da literatura os pontos fortes e limitações de cada uma destas metodologias bem como propor um modelo sinérgico de utilização destas metodologias na fase conceitual do APQP.
\end{abstract}

\section{INTRODUÇÃO}

Lançar produtos que sejam considerados inovadores e disruptivos pelo mercado, antes de todos os concorrentes e, ao mesmo tempo, reduzir o nível de incertezas, aumentando o ganho financeiro frente ao capital investido, é, ou pelo menos deveria ser, o objetivo principal de todas as empresas que investem em processos de inovação.

A indústria automotiva não foge a essa regra, no entanto, não raro, engenheiros e pesquisadores das grandes montadoras de veículos e de seus fornecedores demonstram haver uma grande barreira à inovação dentro de suas empresas. Mesmo possuindo ferramentas consagradas e estabelecidas para este fim e mesmo dizendo, por vezes, haver uma cultura de inovação atrelado a imagem de seus produtos, o que se vê na prática é bem diferente.

É comum haverem menções a plataformas de veículo como sendo totalmente desenvolvidas em solo nacional, no entanto, ao se fazer uma pesquisa mais profunda, o que acaba aparecendo, é que boa parte dos componentes e sistemas utilizados pela engenharia local foram modificados de sistemas já existentes em outras plataformas, ou seja, não passou de uma engenharia de modificação ou adequação ao invés de ser uma engenharia de criação. Isto tem feito com que, cada vez mais, o Brasil seja visto como um grande 'gestor' de projetos que já chegam prontos, enviados pelas casas-matrizes das empresas que atuam no setor.

Dados de empresas de consultoria que atuam no estudo do mercado automotivo global, tais como a PWC [1], apontam que as montadoras de veículos ainda concentram boa parte do investimento em $\mathrm{P} \& \mathrm{D}$ em suas matrizes e que, embora haja uma globalização ou exportação deste investimento desde 2007 até 2015, o mesmo tem sido aplicado em grande escala em países da Ásia como fica demonstrado nos gráficos das Fig.1. 
Fig.1 - Investimentos com $P \& D$ no setor automotivo global (domestico \& exportado)

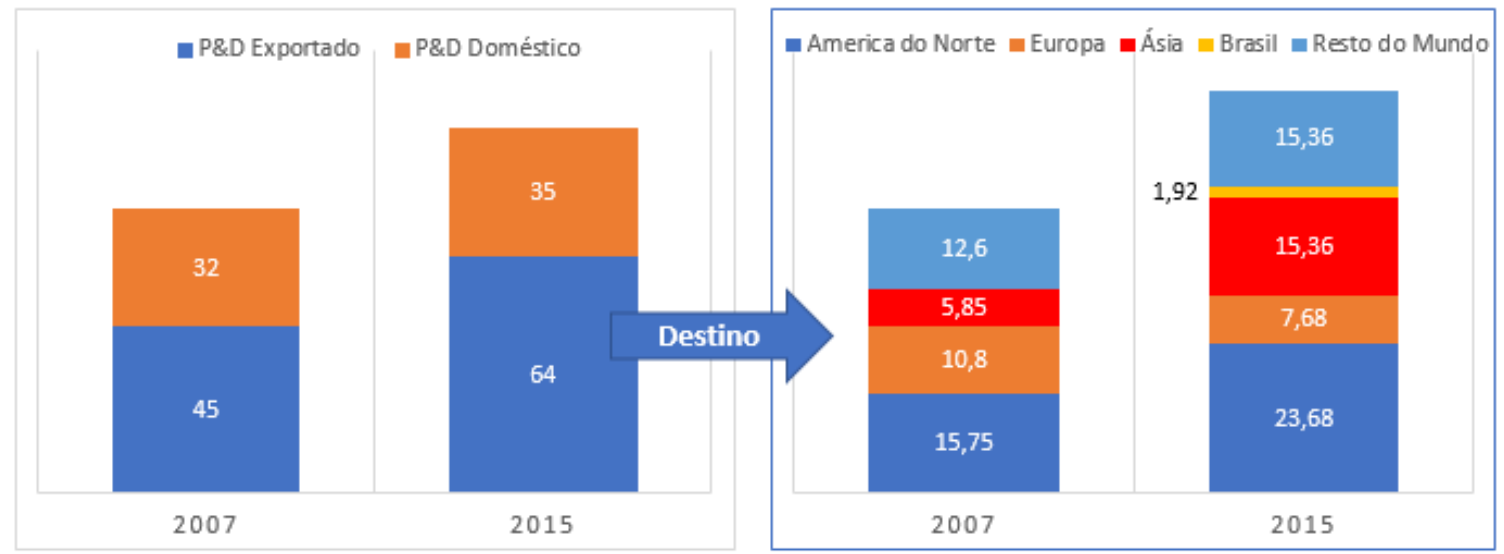

Fonte: PwC Strategy\&: 2015 Global Innovation 1000 Automotive industry findings

Grande parte desta dificuldade está na forma como o setor trabalha novos desenvolvimentos de produtos. Muitas empresas ainda dependem em grande medida de metodologias como o APQP para conduzirem seus PDPs, restringindo, desta forma, novas ideias e oportunidades mais inovadores de serem avaliadas devidamente. Isso faz com que profissionais e empresas do setor fiquem retidas num círculo vicioso de gestão de melhorias incrementais.

É por conta deste contexto que, pesquisas como esta, podem trazer sugestões às OEMs no momento em que as mesmas forem abordar seus projetos ou até mesmo seus respectivos processos de inovação.

\section{UM BREVE REVISÃO DO MODELO APQP}

O APQP - sigla para o termo em inglês Advanced Product Quality Planning ou Planejamento Avançado da Qualidade do Produto desenvolvido e padronizado pela AIAG (Automotive Industry Action Group) [2] - é um processo estruturado para assegurar o desenvolvimento de novos produtos atenda aos padrões de qualidade previamente estabelecidos, garantindo assim a satisfação do usuário final. O processo APQP é descrito no manual 810-358-3003 da AIAG.

Fig. 2 - Fases do APQP

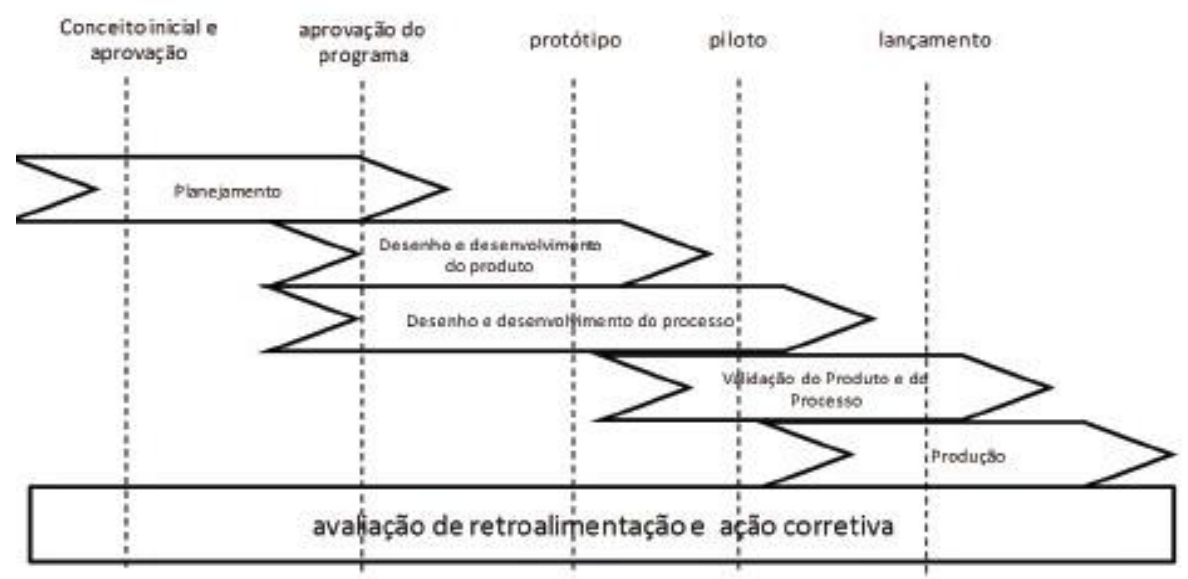

Fonte: AIAG (2008) 
O APQP permite que todos os setores envolvidos no planejamento e desenvolvimento do produto final possam se compreender utilizando uma linguagem comum e ferramentas correspondentes.

De acordo com o Manual de Referência APQP, com a definição da equipe responsável pela condução do programa, a prioridade passa a ser o planejamento, ou seja, estabelecer, através de um cronograma específico, todos os passos necessários para que ocorra êxito, desde o desenvolvimento de um novo produto ou processo até o seu lançamento. São cinco as fases norteadoras do APQP, conforme demonstra a Fig. 2.

Segundo aborda Mello et. Al [3], cada fase é composta por etapas que totalizam quarenta e nove subfases de input e output do controle de qualidade e do desenvolvimento de produtos:

1. Planejar e definir o problema: considera a voz do cliente (através de pesquisa de mercado, informações históricas de garantia e qualidade e as experiências da equipe); plano de negócios e estratégia de marketing; dados de benchmark do produto e do processo; outras premissas do produto e processo envolvendo capacidade técnica; estudo sobre a confiabilidade do produto; inputs do cliente.

2. Verificação do projeto e desenvolvimento do produto: considera os objetivos do projeto; as metas de confiabilidade e de qualidade; lista preliminar de materiais; fluxograma preliminar do projeto; lista preliminar de características especiais de produtos e processo; plano de garantia do produto; suporte da gerência.

3. Verificação do projeto de desenvolvimento do processo: considera Análise de Modo e Efeito de Falha do Projeto (DFMEA); projeto de 'manufaturabilidade' e montagem; verificação do projeto; análises críticas do projeto, construção de protótipo; desenhos de engenharia; especificações de engenharia, alterações de desenho e especificações; requisitos para novos equipamentos, ferramentas e instalações; características especiais de processos; requisitos para meio de medição e equipamento de teste, comprometimento de viabilidade da equipe e suporte da gerência.

4. Validação do produto e processo: considera a corrida piloto de produção; avaliação dos sistemas de medição; estudos preliminares da capacidade do processo; aprovação de peças de produção; testes de validação da produção; avaliação da embalagem; plano de controle da produção; aprovação do planejamento da qualidade e suporte da gerência.

Conforme aborda Lin et. Al [4], de maneira geral, o fluxo de atividades do APQP segue um esquema estruturado conforme ilustra a Fig. 3.

Fig. 3 - Esquema estrutural do APQP

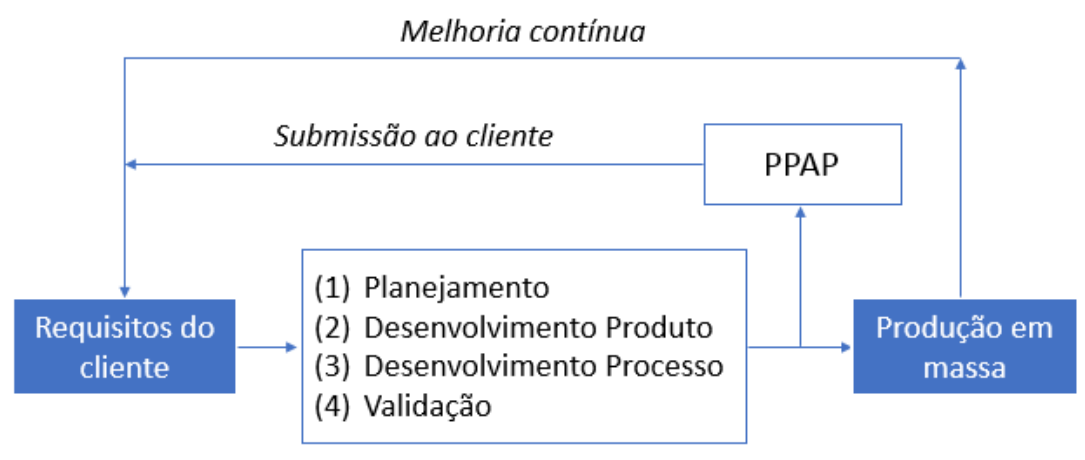

Atividades obrigatórias PPAP:

1. Registro de desenvolvimento

2. Análise de falha do desenvolvimento do produto (DFMEA)

3. Fluxo de manufatura

4. Análise de falha de processo (PFMEA)

5. Plano de controle

6. Análise do sistema de mensuração (MSA)

Requerimentos de dimensionamento

8. Requerimentos de performance

9. Índice de capabilidade do processo (Cpk)

10. Garantia de submissão da peça (PSW)

PILOTO

Fonte: Lin et. al (2009) 
O PPAP - sigla para o termo em inglês Productive Parts Approve Procedure ou Procedimento de Aprovação de Peças Produtivas - é a saída do APQP antes da fase de produção em massa, que geralmente inclui dez atividades obrigatórias. O PPAP deve ser apresentado aos clientes para ter certeza que o produto piloto atende as expectativas dos clientes e aos requerimentos necessários estabelecidos no início do projeto.

O ponto forte desta metodologia está em sua capacidade de, se corretamente aplicada, garantir que os requisitos estabelecidos na origem do projeto sejam plenamente atendidos durante o desenvolvimento do produto e do processo de produção do mesmo, obedecendo tolerâncias aceitáveis ao usuário final.

Com frequência, no entanto, o processo de APQP parte do princípio que os 'Requisitos do cliente` foram devidamente compreendidos e o escopo do problema ou do produto que se quer desenvolver está adequadamente definido. Algo, que na prática, nem sempre ocorre.

Há ainda um ponto negativo nesta metodologia ao não delimitar claramente uma fase conceitual dentro de seu processo de desenvolvimento como outros PDPs clássicos fazem. Isso traz dificuldades ao time para lidar com ideais ou sugestões novas e que pudessem gerar inovações radicais.

\section{FASE CONCEITUAL}

Segundo Clark, Chew e Fujimoto [5] a etapa de criação conceitual de um PDP automotivo deve cobrir desde aspectos como conseguir informações de possíveis usuários finais para serem atendidas durante o desenvolvimento do projeto bem como antecipar necessidades mais gerais ou tendências do mercado.

Para Ibusuki e Kaminski [6], a busca por inovação ainda na fase conceitual de um PDP onde os requerimentos de qualidade, custo e investimento ainda não estão rigidamente delimitados, se constitui em fator potencializador no desenvolvimento de produtos considerados revolucionários ou inovações radicais.

Segundo Rosenfeld et. al [7] entre $80 \%$ a $90 \%$ do custo do produto final é oriundo de decisões tomadas nas fases iniciais de um projeto marcadamente a fase conceitual. A prática ainda mostra que o custo de modificar uma decisão tomada em algum momento de um projeto tende a aumentar exponencialmente ao longo do mesmo trazendo prejuízos e não raro causando atrasos na sua entrega. Ainda segundo o autor, a fase de Projeto Conceitual dentro de um modelo tradicional de PDP é onde ocorre a transição do campo da abstração para a obtenção concreta da possível solução. Rosenfeld et. al [7] esta etapa do PDP é marcada por atividades de busca por informações, representação de possíveis soluções, criação e seleção de alternativas. Estas atividades ocorrem muitas vezes em processos cíclicos e interativos se afunilando até o momento da concepção final do produto.

No caso da metodologia do APQP tradicional, a etapa de conceito não existe por si só mas está compreendida entre as etapas de Planejamento e de Desenvolvimento do Produto conforme ilustra a Fig. 4. 
É dentro destas fases do APQP que as necessidades e desejo do cliente se tornam em requerimentos de projetos e é onde uma definição de conceito de produto é tomada após serem avaliados todos os cenários possíveis.

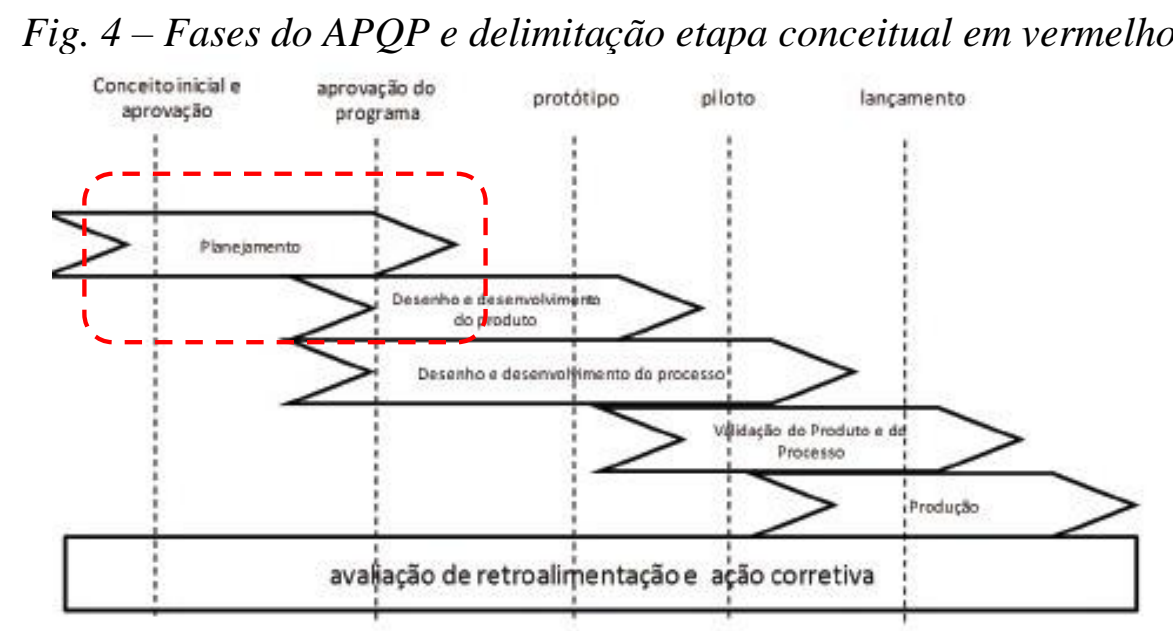

Fonte: AIAG (2008)

É para esta etapa que esta pesquisa se prestará a trabalhar melhorias considerando variáveis de performance de projeto tais como:

- Quantidade de ideias geradas;

- Qualidade das ideias geradas (o quão próximo cada ideia contribui para o conceito final)

- Número de iterações que foram necessárias até a escolha do conceito final;

- Índice de atendimento aos requerimentos

As metodologias do Design Thinking e da TRIZ foram escolhidas para esta pesquisa pois ambas possuem um número grande e diversificado de ferramentas que podem ser utilizadas para suportar esta importante fase do APQP como se procurará demonstrar a seguir.

\section{DESIGN THINKING - DT}

De acordo com Naiman [8], fundadora da Creativity at Work, DT é uma metodologia usada por designers para resolver problemas complexos e encontrar soluções que sejam valorizadas pelo usuário final. Segundo a mesma autora, dentro do DT existe um foco na busca pela solução e não no problema em si. Trata-se de uma metodologia que busca criar o futuro através do uso da lógica, imaginação, intuição sem abrir mão do pragmatismo e da sistemática na exploração de possibilidades e na obtenção dos resultados esperados.

De acordo com Dorst [9], DT também pode ser tratada como uma forma de abordar problemas e apresentar soluções de uma maneira mais abrangente do que aquelas tradicionalmente utilizadas por designers dentro de seu campo de atuação podendo fazer uso das mais diferentes áreas do conhecimento humano tais como as ciências sociais e a administração. Brown [10] aborda a utilização do DT dentro da área de negócios como suporte à capacidade criativa conjuntamente com outros métodos auxiliares para identificar adequadamente as necessidades do usuário final e enquadrá-las dentro daquilo que é tecnicamente viável, criando assim, uma oportunidade de negócios conforme ilustra a Fig. 5. Ainda segundo o mesmo autor, o DT trata-se de um método que permeia as atividades de inovação com foco no ser humano, através de um processo de compreensão detalhado que 
ocorre por meio de observações diretas com a finalidade de extrair os desejos e necessidades das pessoas muitas vezes não explicitados dentro de uma pesquisa ou entrevista tradicionais.

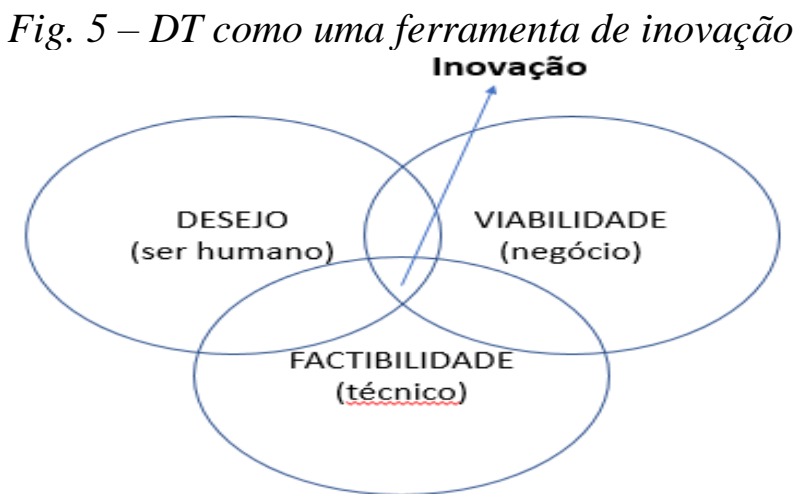

Fonte: IDEO U LLC (2017)

De acordo com Robson [11], ao contrário do processo analítico convencional, o DT inclui uma etapa preliminar de geração intensiva de ideias durante uma seção de brainstorming. Esta parte do DT tem sido reconhecida como de grande importância pois ela traz à tona elementos muitas vezes ignorados assim como ambiguidades e falsas hipóteses feitas anteriormente.

Para Cohen [12] uma grande parte do sucesso do DT está baseado na empatia entre o designer e o usuário final que se beneficiará do produto que está sendo desenvolvido. Faz parte do DT incluir uma série de atividades de checagem junto ao usuário final para saber se a ideia ou conceito beneficia e traz valor de fato ao usuário atendendo suas demandas de maneira geral.

Fig. 6 - Etapas do DT estabelecidas pela Design Council (Reino Unido)

O que e por que?

Como

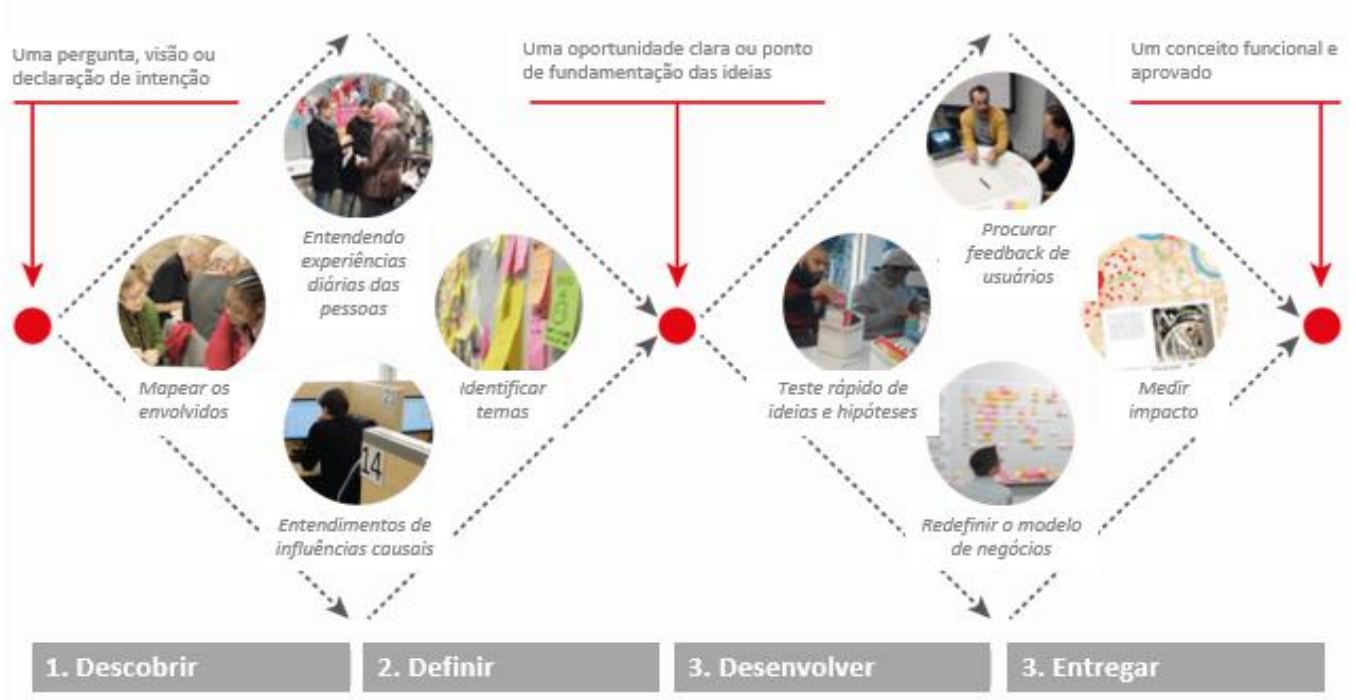

De acordo com Naiman [8] o ciclo do DT envolve intensa observação para descobrir necessidades do usuário final dentro do contexto e das dificuldades reais de uma situação particular para que ideias criativas sejam geradas, testadas e refinadas até que se tenha atingido a solução final. A Design Council que fica na Inglaterra procura descrever o DT num processo de 4 fases distintas: descoberta, definição, desenvolvimento e entrega. Todas estas 
etapas fluem em etapas cíclicas de divergência e convergência até que o conceito final seja encontrado conforme ilustra a Fig. 6.

Para Plattner et. al [13], o DT possui seis estágios conforme mostra a Fig. 7. Segundo o mesmo autor, as etapas não são lineares e podem ocorrer de maneira cíclica.

Fig. 7 - Etapas do design thinking na HPI D-School

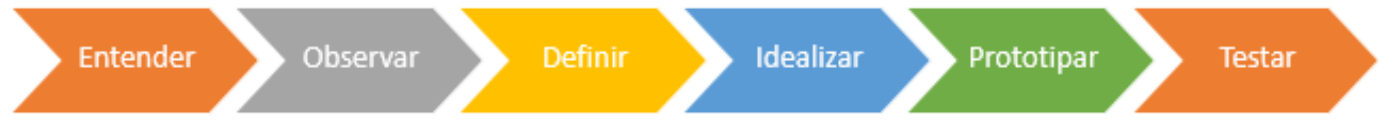

Fonte: Plattner et. al (2009)

Para Mueller e Thoring [14], as etapas acima se desdobram da seguinte maneira:

- Entender: busca de informações existentes sobre o assunto ou problema que se queira tratar. É feita em grande medida através de pesquisa secundária;

- Observar: o objetivo desta etapa é obter informações a respeito do problema e das necessidades não percebidas do usuário final;

- Definir: as conclusões obtidas através das etapas anteriores são então compiladas de maneira visual através de utilização de storytelling criando assim uma microteoria sobre o problema através da ótica do usuário final que pode ser chamada de POV - Point of View.

- Idealizar: esta fase se inicia com uma atividade de geração de ideias utilizando ferramentas como o Brainstorming para trazer soluções ao POV levantado na etapa anterior e, posteriormente, uma atividade de priorização é feita para elencar as melhores ideias que serão levadas para a próxima fase;

- Prototipar: na fase de prototipagem, as ideias selecionadas na etapa anterior são prototipadas de maneira rápida.

- Teste: posteriormente o protótipo é levado para a avaliação do usuário final que poderá então validar ou trazer novas melhorias ao conceito proposto pelo time do projeto.

\section{TRIZ}

Segundo relata o Instituto Altshuller de Estudos da TRIZ [15], "TRIZ" vem das palavras russas para "teoria da resolução de problemas inventivos". Segundo reporta o mesmo instituto, G.S. Altshuller e seus colegas na antiga U.R.S.S. desenvolveram o método entre 1946 e 1985 após analisarem mais de 40.000 patentes e estabelecerem padrões, com base nestas patentes, de como os problemas eram resolvidos e novas invenções realizadas.

Para o próprio Altshuller [16] a TRIZ é um método de resolução de problemas com base na lógica e em dados, não na intuição, o que acelera a capacidade da equipe do projeto na resolução destes problemas de maneira criativa. Para o autor, a TRIZ é apresentada como um método que proporciona repetitividade, previsibilidade e confiabilidade devido a sua estrutura e abordagem algorítmica.

Para Savransksy [17], TRIZ é uma metodologia sistemática baseada no conhecimento obtido na resolução prévia de problemas inventivos.

Para Zlotin et. al [18] o uso da TRIZ não ficou concentrado apenas em áreas técnicas, mas também migrou para outras áreas tais como administração, política e publicidade sendo, 
inclusive, utilizada no gerenciamento de projetos, em sistemas de gestão de risco assim como em iniciativas de inovação organizacional.

Para Gadd [19] a TRIZ consiste numa caixa de ferramentas contendo métodos que cobrem todos os aspectos da compreensão de um problema e de sua resolução.

Para Ilevbare et. al [20], os benefícios da metodologia — sua capacidade de produzir ideias inovadoras e soluções - permanecem proeminentes e são amplamente aceitas. Segundo os autores, organizações interessadas em ultrapassar os limites da inovação, permanecendo competitivas, devem considerar esta abordagem se tiverem os meios e a paciência para compreendê-la e incorporá-la em suas estratégias de processos de inovação.

Existem diversas formas de organizar as ferramentas e técnicas utilizadas na TRIZ. Um fluxograma pode ser bastante útil pois, a partir dele, pode-se ter uma ideia de como as ferramentas estão relacionadas bem como o papel que cada uma delas possui dentro do fluxo geral da TRIZ. Para este fim utilizaremos o modelo desenvolvido por Domb e Dettmer [21]. A Fig. 8 representa este modelo da TRIZ para o desenvolvimento de produtos.

Fig. 8-Fluxo geral da TRIZ mostrando algumas de suas ferramentas

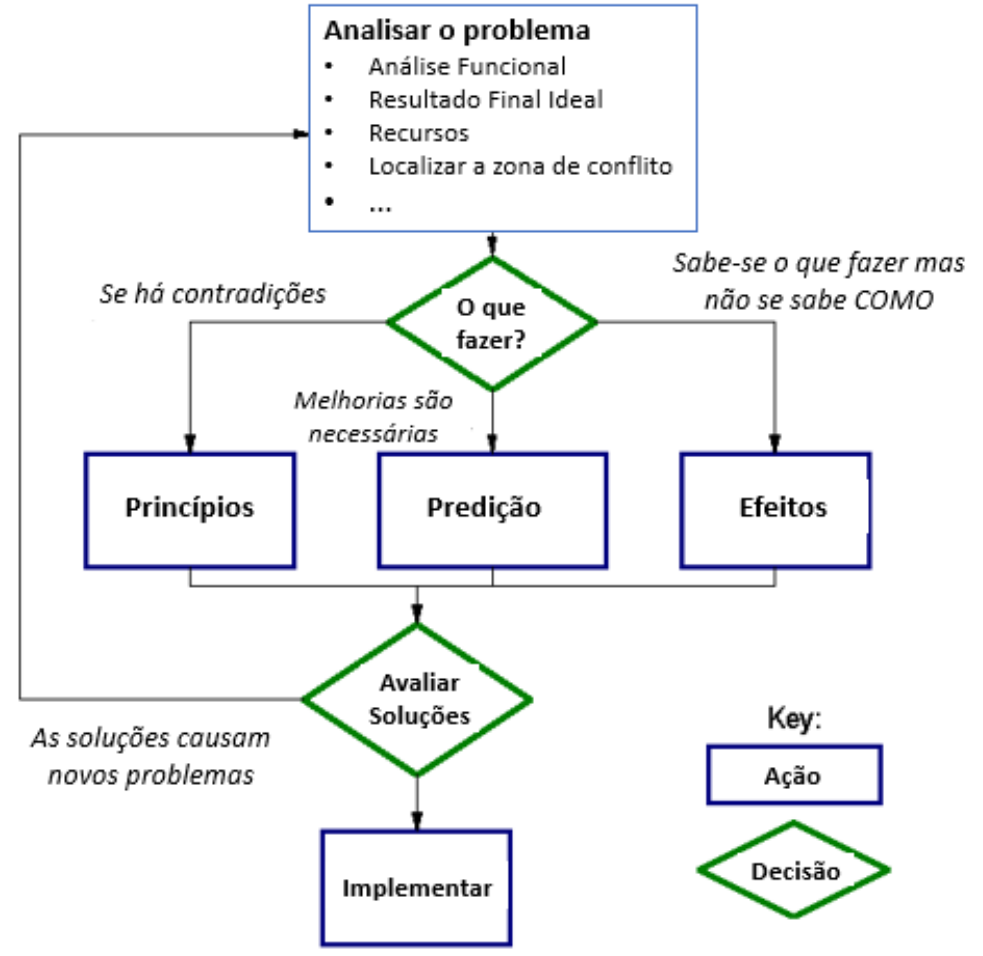

Fonte: adaptado Domb e. Dettmer (1999)

Segundo Domb e Dettmer [21], não são mostrados no fluxograma acima a análise 'S-Field' (um sistema de modelagem diagramática usado para descrever problemas e identificar categorias de soluções - geralmente usada para problemas difíceis ou avançados) e a 'ARIZ' que pode ser traduzido como "algoritmo para resolver problema inventivo" (trata-se de um algoritmo não-computacional composto por uma série de mais de 70 perguntas sendo uma forma alternativa de vincular as ferramentas e técnicas de TRIZ). 
Conforme mostra a Fig. 8 a primeira fase da TRIZ é a de 'Análise do Problema'.

Análise funcional - analisa o sistema, subsistemas e componentes em termos de funções desempenhadas. Uma técnica mais recente utilizada em TRIZ é conhecida como "trimming" (examina cada função para ver se de fato ela é necessária e, em sendo, se há algum outro elemento do sistema que poderia executar a mesma função).

Resultado final ideal - expressa a situação em termos de por que a inovação é necessária e qual seria o melhor resultado possível que se poderia obter na resolução de um dado problema sem, no entanto, considerar limitações tecnológicas ou de implementação para o atingimento deste resultado. O progresso obtido em um projeto em função do resultado ideal é chamado de "idealidade" e é definido pela seguinte equação:

$$
\text { Idealidade }=\frac{\sum \text { Benefícios }}{\left(\sum \text { Custos }+\sum \text { Prejuízos }\right)}
$$

Análise de recursos - identificação de todos os recursos, fontes de energia, objetos disponíveis, informações, funções e outros elementos que estão dentro ou perto do sistema e que poderiam ser combinados com os elementos dentro do sistema para melhorá-lo.

Identificando as zonas de conflito - trata-se da compreensão da causa exata do problema. A "zona" refere-se ao tempo e lugar em que o problema ocorre. O diagrama de resolução de conflito (CRD) é uma ferramenta eficiente para localizar as zonas de conflito.

Ainda segundo Domb e Dettmer [21], em cerca de $45 \%$ dos problemas a resolução se torna óbvia logo após a conclusão da fase de análise. Neste caso é comum ir direto para a fase de implementação. Por outro lado, segundo os autores, se o problema não foi resolvido ou se há uma solução alternativa que traga maior eficiência, parte-se então para a utilização das ferramentas do banco de dados da TRIZ: Princípios, Previsões e Efeitos. Neste caso, pode-se optar por utilizar apenas algumas das ferramentas ou todas elas, sendo cada uma das ferramentas descritas na sequência.

Princípios (também chamados de resolução de contradições) - contradições técnicas ocorrem quando o estado desejado não pode ser alcançado porque alguma coisa no sistema impede. Contradições físicas são situações onde um objeto possui requisitos contraditórios. Uma vez que a contradição é definida em termos de seus parâmetros padrão, o problema é resolvido através da aplicação dos quatro princípios da separação (contradições físicas) ou dos quarenta princípios de resolução de conflitos (contradições técnicas). A base de dados destes princípios está disponível em várias formas e pode ser baixada da web em http://www.trizjournal.com.

Predição (também chamado de previsão de tecnologia) - a TRIZ identificou oito padrões de evolução técnica que um sistema pode demonstrar ao longo do tempo. Projetos de sistemas, subsistemas ou componentes podem ser movidos para o próximo estágio dentro de um padrão específico, uma vez que o padrão é identificado. Os oito padrões são:

1. Aumento da idealidade;

2. Fases de evolução;

3. Desenvolvimento não uniforme de elementos do sistema;

4. Maior dinamismo e controle;

5. Complexidade crescente seguida de simplicidade; 
6. Encontro e desencontro de partes;

7. Transição para o nível micro e uso de campos;

8. Diminuição da interação humana (automação crescente).

Efeitos - usa a fenomenologia científica e de engenharia e seus efeitos fora da disciplina em que foram desenvolvidas originalmente. As ferramentas incluem bases de dados, enciclopédias de ciências e pesquisas da literatura técnica para encontrar formas alternativas para alcançar as funções que são necessários para resolver o problema.

Ainda segundo Domb e Dettmer [21], último bloco no fluxograma é a fase de 'avaliação das soluções'. Nesta etapa as possíveis soluções são comparadas com o 'resultado final ideal' e certifica-se de que as melhorias trarão avanços atendendo as necessidades do usuário final. Ainda conforme os autores, a vantagem da TRIZ é que as iterações são muito rápidas e um grande número de ideias inovadoras é desenvolvido em cada etapa.

Zlotin et al. [18] classificam as ferramentas em três grupos: ferramentas de análise, ferramentas baseadas em conhecimento e operadores psicológicos. Ferramentas analíticas como análise 'Su-Field', análise funcional e o algoritmo ARIZ ajudam a definir, formular e modelar um problema, enquanto as ferramentas de conhecimento, como os 40 princípios inventivos, 76 soluções-padrão e efeitos fornecem recomendações para a transformação do sistema (ou seja, resolução de problemas). Os operadores psicológicos ajudam a facilitar processo criativo de resolução de problemas.

Para Gadd [19], a TRIZ possui considerável vantagem sobre outros métodos aplicados à resolução de problemas inventivos. Métodos como brainstorming, mapeamento da mente, pensamento lateral, análise morfológica, etc., têm a capacidade de identificar ou revelar um problema e suas causas, mas não possuem a capacidade de realmente apontar soluções para o problema.

Fig. 9 - Processo sistemático de resolução de problemas da TRIZ

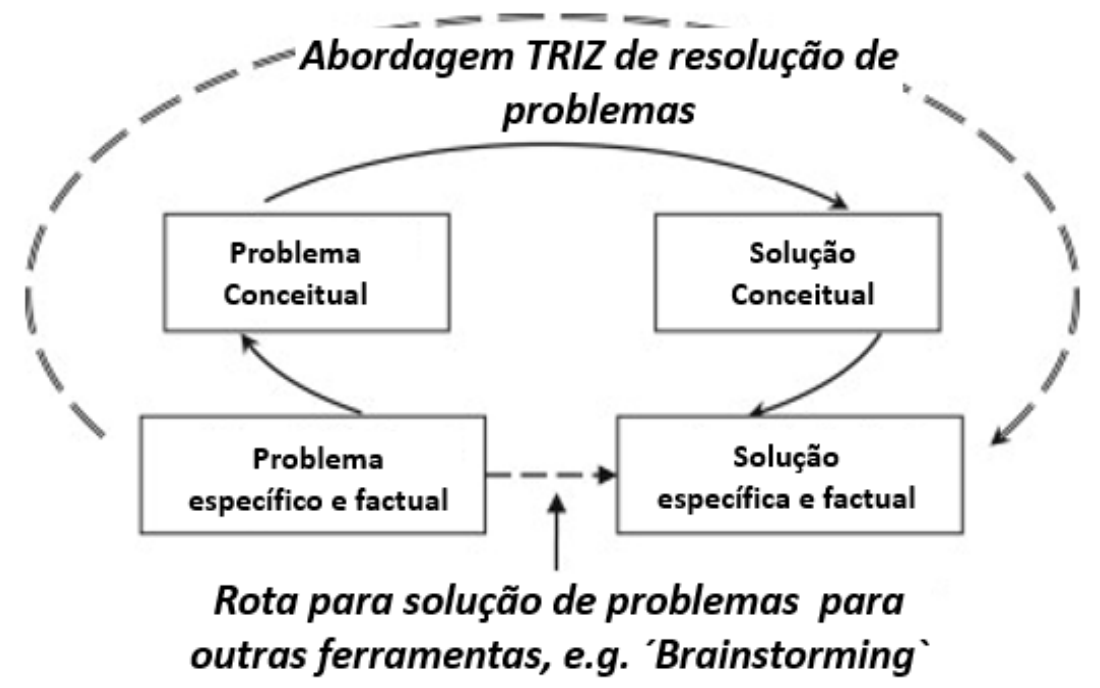

Fonte: adaptado Savransky (2000) e Gadd (2011)

Ainda para Gadd [19], uma característica central à TRIZ é o conjunto de soluções conceituais para problemas técnicos que ela categoriza e armazena. Segundo o mesmo autor, para aplicar 
qualquer uma dessas soluções, um problema técnico específico e factual precisa ser reduzido a seus fundamentos e descrito num formato conceitual como mostra a Fig. 9.

\section{SINERGIA ENTRE DT E TRIZ}

Analisando-se ambas as metodologias comparativamente, pode-se concluir que existem similaridades entre as mesmas tais como:

- Possuem um processo estruturado e que pode se adaptar a situações específicas;

- Dão grande ênfase à parte analítica de 'definição do problema' delegando o sucesso do projeto a esta etapa;

- Permitem ciclos de iterações que culminam em geração de novas ideias até que se chegue numa proposta de solução;

- Têm potencial de gerar invenções ou inovações consideradas radicais ou disrupitivas;

Para Hentschel et. al [22], ao mesmo tempo as metodologias possuem similaridades, ambos os modelos também se complementam conforme ilustra a Tab. 1 a seguir.

Tab.1-Características complementares da DT e da TRIZ

\begin{tabular}{lll} 
& \multicolumn{1}{c}{ DT } & \multicolumn{1}{c}{ TRIZ } \\
\hline Base do Processo & Baseado na criatividade & Baseado em dados e conhecimento \\
\hline Aprendizagem / Aplicação & É fácil e amigável & Complexa e de difícil aplicação \\
\hline Foco & No usuário & No sistema técnico \\
\hline Resultado esperado & $\begin{array}{l}\text { Dar o máximo de } \\
\text { satisfação aos usuários }\end{array}$ & Idealidade do sistema técnico \\
\hline Afinidade & Pessoas intuitivas & Pessoas analíticas \\
\hline
\end{tabular}

Fonte: adaptado de Hentschel et al. (2013)

Para o mesmo autor, quando se trata de TRIZ, há uma percepção de estar lidando com ferramentas altamente sofisticadas e um processo de aprendizagem muito demorado. Como proposta, os autores propõem a facilitação a compreensão da TRIZ pela utilização do DT como elemento mediador. Para os autores, a DT permite facilmente estabelecer um processo de inovação sistemática de maneira mais amigável ao mesmo tempo que abre caminho para uma maior aceitação e uma compreensão mais profunda da TRIZ.

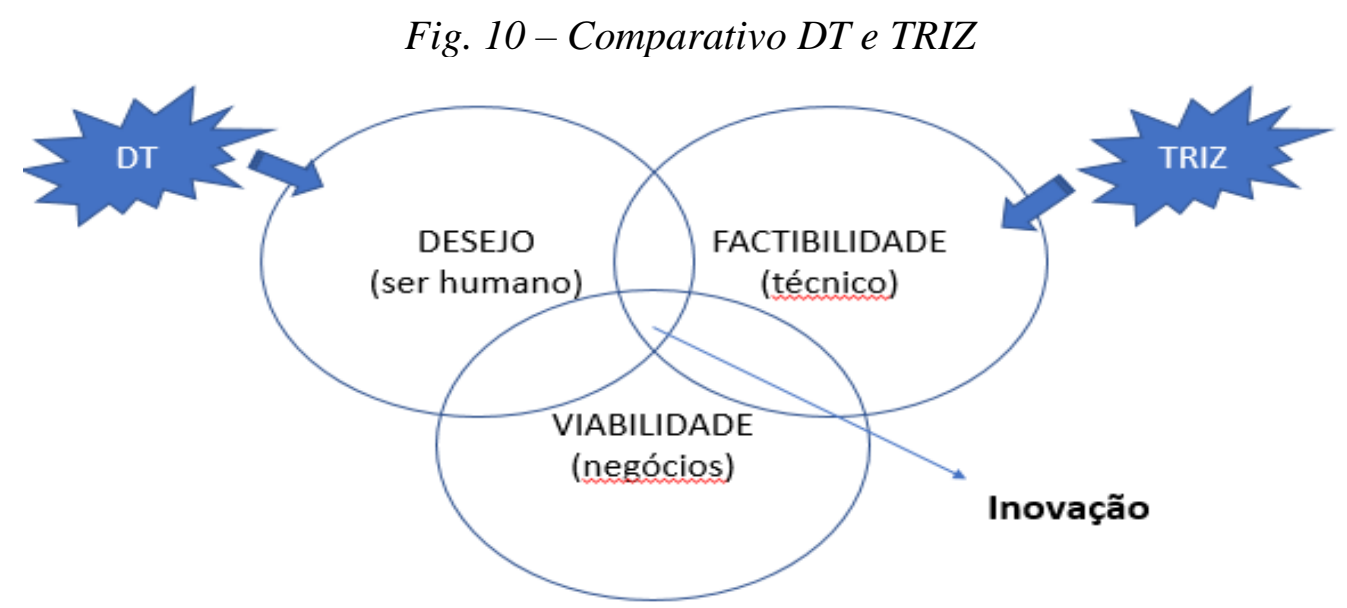

Fonte: adaptado de Hentschel et al. (2013) 
Uma das formas de utilização conjunta destas metodologias e que se quer sugerir através deste trabalho é a adoção do DT como modelo principal ou modelo base, no entanto, auxiliado pelas ferramentas da TRIZ.

Este suporte da TRIZ à DT pode ocorrer justamente na etapa onde a DT mais carece de uma abordagem analítica e técnica que é dentro da sua fase de geração de ideias ou também chamada de fase 'ideação` conforme mostra a Fig. 11.

Fig. 11 - Processo DT com o suporte da TRIZ

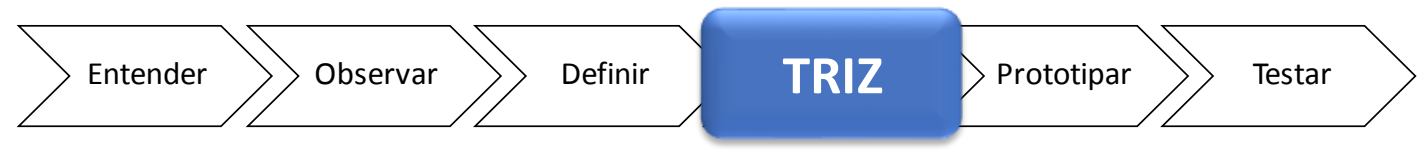

No momento em o DT identifica o problema e passa a idealizar ou a gerar ideias dentro de um processo livre e auxiliado pelo Brainstorming é que muitos erros podem ser gerados. Não raro há uma elevada quantidade de ideias de baixa qualidade levando a um alto grau de iterações dentro da fase inicial do PDP até que haja uma depuração das ideias ou até que se chegue a um consenso sobre o melhor conceito a ser levado às demais fases do projeto de desenvolvimento.

A ideia por trás do Brainstorming é que o julgamento está suspenso e não há ideia que seja considerada ruim. Isso incentiva as pessoas a falar sobre tudo o que vem à mente gerando, por vezes, uma quantidade muito grande de ideias. Quando o julgamento está suspenso o grupo pode ir rapidamente por um caminho cheio de ideias irrelevantes ou irrealistas gerando desperdício de tempo e inúmeros retrabalhos.

Como mencionado, a metodologia TRIZ apresenta uma abordagem mais técnica e analítica. Dentro desta metodologia encontramos ferramentas que podem ajudar na correta análise e definição do problema como por exemplo a Análise Funcional e a definição do Resultado Ideal esperado. Há ainda um grupo de ferramentais sequenciais para o tratamento do problema até que se encontre uma solução, seja pela utilização dos Princípios de Engenharia ou pela Predição conforme ficou demonstrado na Fig. 8.

Desta forma, a combinação dos modelos da DT e da TRIZ como proposto na Fig. 11, se aplicados corretamente à fase inicial de um APQP, podem trazer incrementos reais de qualidade e assertividade a projetos de desenvolvimento de novos produtos, bem como aumentar as chances de que inovações mais radicais ocorram, deixando de ser assim apenas um processo para garantir a qualidade do produto final.

\section{CONCLUSÃO}

Este trabalho procurou entender os principais pontos positivos e negativos das metodologias do DT e da TRIZ através de uma ampla pesquisa bibliográfica ao mesmo tempo que propõe uma forma de interação entre as mesmas com o objetivo de gerar um modelo mais robusto que pudesse ser utilizado como suporte à fase conceitual do processo APQP. 
Conforme foi visto, a metodologia combinada da DT e TRIZ, se adequadamente aplicada na fase inicial do processo APQP, pode trazer melhorias na prospecção do problema e na sua correta definição. Pode ainda levar mais rapidamente o time do projeto ao conceito de produto ou da solução final e que será encaminhando às demais fases do APQP.

Conclui-se, portanto, que ambas as metodologias são sinérgicas e podem suportar o processo de APQP na indústria automotiva.

\section{REFERÊNCIAS}

[1] JARUZELSKI, B.; HIRSH, E. R. The 2015 global innovation 1000: automotive industry findings. PwC Strategy \&, 2016. Disponível em https://www.strategyand.pwc.com/reports/2015-global-innovation-1000-auto-industry.

Acesso em 20 mai. 2017.

[2] AUTOMOTIVE INTERNATIONAL ACTION GROUP. Advanced product quality planning (APQP) and control plan. Automotive Industry Action Group, 2008.

[3] MELlO, E. B.; GANZER, P.P.; RASIA, I. C. R. B.; OLEA, P. M.; ROCHA, J. M. Processo de desenvolvimento de produtos e o sistema Stage-Gate. Porto Alegre: Gestão Contemporânea, 2012.

[4] LIN, L.; LI, T.; KIANG, J. P. A continual improvement framework with integration of CMMI and Six-sigma model for auto industry. Qual. Reliab. Engng. Int., V. 25, pp. 551$569,2009$.

[5] CLARK, K. B.; CHEW, W. B.; FUJIMOTO, T. Product development in the world auto industry: strategy, organization and performance. Brookings Papers on Economic Activity, V. 1987, n³, pp. 729-771, 1987.

[6] IBUSUKI, U.; KAMINSKI, P. C. Product development process with focus on value engineering and target-costing: a case study in an automotive company. Int. J. Production Economics, São Paulo, V. 105, pp. 459-474, 2007.

[7] ROZENFELD, H.; FORCELliNI, F. A..; AMARAL, D. C.; TOLEDO, J. C.; SILVA, S. L.; ALLIPRANDINI, D. H.; SCALICE R. K. Gestão de desenvolvimento de produtos: Uma referência para melhoria de processo. São Paulo: Saraiva, 2006.

[8] NAIMAN, L. Design thinking as a strategy for innovation. Disponível em http://www.creativityatwork.com/design-thinking-strategy-for-innovation. Acesso em 05 fev. 2017.

[9] DORST, K. Frame Innovation: create new thinking by design. Cambridge, MA: MIT Press, 2015.

[10] BROWN, T. Design Thinking. Disponível em https://hbr.org/2008/06/design-thinking. Acesso em 14 jan. 2016.

[11] ROBSON, M. Brainstorming problem-solving in groups. Burlington, VT: Gower Publishing Company, 2002.

[12] COHEN, R. Design Thinking: a unified framework for innovation. Disponível em http://www.forbes.com/sites/reuvencohen/2014/03/31/design-thinking-a-unified-frameworkfor-innovation/\#25f72c6756fc. Acesso em 05 fev. 2017.

[13] PLATTNER, H.; MEINEL, C.; LEIFER, L. J. Design Thinking: understand, improve, apply. Berlin: Springer-Verlag, 2011.

[14] MULLER, R. M.; THORING, K. Understanding Design Thinking: a process model based on method engineering. Disponível em https://www.designsociety.org/publication/30932/understanding_design_thinking_a_process model_based_on_method_engineering. Acesso em 11 fev. 2016. 
[15] ALTSHULLER INSTITUTE FOR TRIZ STUDIES. What is TRIZ? The Altshuller Institute for TRIZ Studies. Disponível em: http://www.aitriz.org/triz. Acesso em 19 fev. 2017. [16] ALTSHULLER, G. Creativity as an exact science. New York, NY: Gordon \& Breach, 1984.

[17] SAVRANSKY, S. D. Engineering of creativity: introduction to TRIZ methodology of inventive problem solving. Boca Raton, FL: CRC Press, 2000.

[18] ZLOTIN, B.; ZUSMAN, A.; KAPLAN, L.; VISNEPOLSCHI, S.; PROSEANIC, V.; MALKIN, S. TRIZ Beyond technology: the theory and practice of applying TRIZ to nontechnical áreas. Disponível em https://triz-journal.com/triz-beyond-technology-the-theoryand-practice-of-applying-triz-to-non-technical-areas. Acesso em 19 fev. 2017.

[19] GADD, K. TRIZ for engineers. West Sussex, UK: John Wiley \& Sons, 2011

[20] ILEVBARE, I. M.; PROBERT, D.; PHAAL, R. A review of TRIZ, and its benefits and challenges in practice. Technovation, London, UK, V. 33, n²-3, pp. 30-37, 2013.

[21] DOMB, E.; DETTMER, H.W. Breakthrough innovation in conflict resolution: marrying TRIZ and the thinking process. Disponível em https://triz-journal.com/breakthroughinnovation-conflict-resolution-marrying-triz-thinking-process. Acesso em 20 fev. 2017.

[22] HENTSCHEL, C.; CZINKI, A. Design thinking as a door-opener for TRIZ: paving the way towards systematic innovation. Disponível em http://home.htwberlin.de/ hentc/Handouts/Veroeffentlichungen/TFC2013_Hentschel_Czinki. pdf. Acesso em 22 mai. 2017. 